\title{
Research and Design of Hydraulic AGC System Model of Cold Rolling Mill
}

\author{
Yuheng Yin ${ }^{1, *}$,Liwei $\mathrm{Cao}^{2}$,Yuran Wang ${ }^{1}$ and Hang $\mathrm{Fu}^{1}$ \\ ${ }^{1}$ School of Automation, Harbin University of Science and Technology,Harbin,China \\ ${ }^{2}$ department of production coordination, Daqing Petrochemical Engineering Co. Ltd, \\ Daqing, China \\ *E-mail :13503636310@163.com
}

\begin{abstract}
The dynamic analysis model establish of Cold Rolling Mill Hydraulic AGC System can fully and easily analyze the various factors of rolling process on the final thickness control and rolled accuracy. The simulation of the hydraulic AGC system can give out the structure and performance of the system, determining a greater impact on system performance parameters for the rolling of new varieties to provide reliable evidence and arguments, while the mill mechanical and hydraulic system fault diagnosis has certain reference value. Adaptive control to improve the process of rolling mill rolls unsteady exit thickness accuracy can play a big role, but due to many reasons, the traditional adaptive control methods in the actual mill is difficult to achieve.
\end{abstract}

Keywords: Cold rolling mill; Thickness control; Simulation analysis

\section{Introduction}

Sheet and strip thickness accuracy is an important quality indicator, thickness control is the strip rolling in the field of key technologies. Automatic thickness control system AGC (short for Automatic Gauge Control) is used to continuously measure the actual thickness of the strip through the thickness or the sensor, according to deviation signal of the measured value comparing with a given value, by controlling circuit and a device or function of a computer program, and change the depressed position, the tension or the rolling speed, the thickness deviation will be controlled under the permissible range.

The number of cold rolling machine at home and abroad is large, mathematical models used during control process are different. These models in the in various mills have been used, which model is more suitable for the specific rolling mill has no comparison. Exploring for one which is more suitable for the mathematical model and process control model provide a reliable basis for the further transformation of the mill, rolling new products, improve product yield, quality and variety.

\section{A. Forward feedback AGC}

The initial thickness of incoming materials $h_{\text {Oact }}$ can be obtained from the entrance thickness gauge, the entrance set thickness of strip steel is $h_{\text {Oset }}$, and the thickness difference is calculated by the difference of the two values $\delta h_{o}$. The screw down position should be changed according to actual $\delta h_{o}$, and when this strip steel is put in the deformation area, the 
impact of the thickness difference to the exit thickness can be eliminated according to the calculated screw down adjustment.

And the same as second flow AGC, while adjusting pressure, we must make compensation for the roll on the rack rate of the pre-S to ensure constant tension of the front rack.

\section{B. Monitoring AGC}

Monitoring AGC is applied to control the position of the hydraulic cylinder, speed of roller, speed of first roller, according to the thickness difference obtained from entrance gauge to change the roll gap and constant tensile force to eliminate thickness deviation.

Because existing a time lag of monitor AGC, it is difficult to correct mutations caused due to entry thickness after rolling thickness deviation, it can only be used to correct the LF, slowly varying bias. Rolling thickness deviation which is caused by the roll of thermal expansion and friction caused by the changed slowly interference amount, not perfectly linear of elasticity curve, the inaccurate of set value of no-load and the rolling pressure roll, the displacement sensor and the pressure sensor measurement errors caused, can be compensated for with using monitor AGC.

\section{Flow control per second AGC}

There can be a small delay after the actual measurement of entrance thickness of strip steel $h_{\text {Oact }}$. $V_{0}$ (Entrance speed)and $V_{1}$ (exit speed)within the deformation area are obtained, and the exit thickness $h_{1 \text { cal }}$ can be accurately calculated from the constant theory of flow per second in the deformation area. Exit value is set as $h_{1 \text { set }}$, then the thickness difference generated is $\delta h=h_{1 c a l}-h_{1 s e t}$. According to the difference obtained above, appropriate adjustment of screw down position is done to ensure a constant exit thickness.

Adjusting the pressure will inevitably lead to changes in the tension before the rack, in order to maintain constant tension, we must adjust the frame rate of the pre-S rollers, to compensate the $\mathrm{S}$ roller speed.

\section{Film Thickness and Roll Eccentricity Compensation}

From the wear rate accelerated to a steady speed with a rolling speed and decelerate to cast steel tail speed, because the speed changed greatly, causing oil film bearing oil film thickness variations leaving the roll gap transformation occurs, leading to the strip exit thickness variation, this the first frame has a film thickness compensation.

Because of the high precision for thickness of cold rolling, the influence of roll eccentricity can not be ignored, so the first frame which angle measurement device has been positioned used to make the eccentricity compensation depending on the position.

\section{Basic Theory on Thickness Control}

\section{A. The Mill Bounce Equation}

In the production of rolling, the interaction force between rolling rolls and the rolling is applied through rolling force, the rolling member is plastically deformed so that the thickness of rolling is reduced, which is one of the main purposes during the rolling process. At the same time, rolling rolls gave the rolling rolls the same reaction force, each of the base parts has a certain elastic deformation, and the cumulative effects of the elastic deformation are reflected with the increase in the roll nip, this is called for the bounce. 
Mill stretch is generally up to $2 \sim 5 \mathrm{~mm}$, the cold-rolled sheet, because of the small reduction, bouncing effects must be considered, and the bounce value should be accurately calculated.

Mill operation can only adjust the roll gap $\mathrm{S}_{0}$, light control system to complete the task is how to adjust the no-load roll gap to achieve the required thickness, and the bounce phenomenon can be used in the mill bounce equation describing:

$$
h=S+\frac{P-P_{0}}{K_{m}}+S_{F}+G+O
$$

Type of h-rolled piece exit thickness (mm); S-roll gap location, mm; SF-roll gap caused by the change of roll bending, $\mathrm{mm}$;

P-rolling force, KN; Km-mill longitudinal stiffness, $\mathrm{kN} / \mathrm{mm}$; O to the oil film thickness, $\mathrm{mm}$; G-roll gap zero compensation, mm.

Mill stretch curve is shown in Figure 1, the tests showed that the rolling mill spring curve is not linear in the small rolling force curve area, until the rolling force increased to a certain value, the rolling force and the deformation will present linear relationship. This phenomenon can be explained with using deformation and the bearing clearance. The nonlinear region is not stable if each roll has been changed.

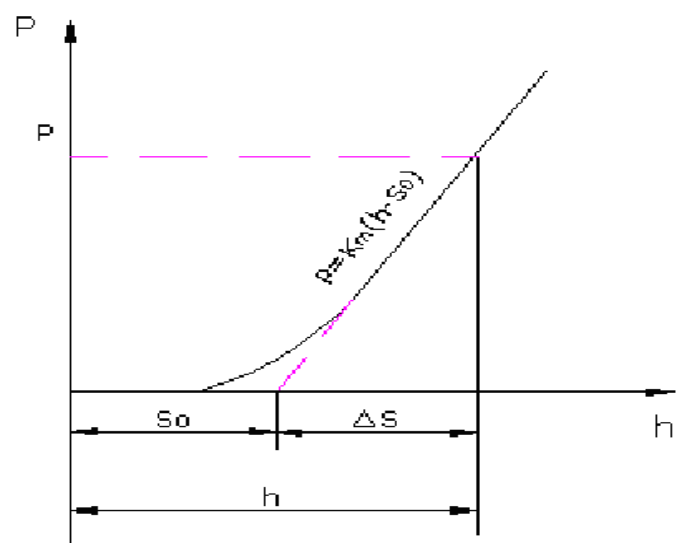

Figure 1. Mill Stretch Curve

\section{B. Thickness control equation of rolling mill}

Equation (1) show the influence of thickness of the rolling force in rolling out, all kinds of process parameters such as hardness, $\mathrm{K}, \mathrm{H}$, etc. The influence of material thickness is by rolling force change and influence of $\mathrm{H} \mathrm{P}$. And the rolling force formula is a nonlinear function, namely

$$
P=f\left(H, h, \mu, \tau_{b}, \tau_{f}, K\right)
$$

Type of H-mill entry thickness of rolled piece, mm; Mu, friction coefficient of rollers and rolled piece; Tau b-after tensile stress, MPa; Tau f-before tensile stress, MPa; K-strip hardness, MPa.

Incremental form of equation can be described as follows:

$$
\delta P=\frac{\partial P}{\partial H} \delta H+\frac{\partial P}{\partial h} \delta h+\frac{\partial P}{\partial \tau_{b}} \delta \tau_{b}+\frac{\partial P}{\partial \tau_{f}} \delta \tau_{f}+\frac{\partial P}{\partial K} \delta K+\frac{\partial P}{\partial \mu} \delta \mu
$$


When taking the slow change in the thickness of oil film and the zero drift of roll gap into consideration, spring equation can be described as incremental form:

$$
\delta h=\delta S+\frac{\delta P}{K_{m}}+\delta S_{F}
$$

Substitute formula (1) in the spring equation of formula (2) to obtain the exitthickness control equation:

$$
\delta h=\frac{\left(\frac{\partial P}{\partial H} \delta H+\frac{\partial P}{\partial K} \delta K+\frac{\partial P}{\partial \mu} \delta \mu\right)+\left(\frac{\partial P}{\partial \tau_{b}} \delta \tau_{b}+\frac{\partial P}{\partial \tau_{f}} \delta \tau_{f}\right)+\left(K_{m} \delta S+K_{m} \delta S_{F}\right)}{K_{m}+Q}
$$

The above formula shows intuitively control the amount $\delta S, \delta S_{F}$, disturbance $\delta H, \delta K$, $\delta \mu$ and other factors may affect the objective function $\delta h$.

When $H, K, \mu, \tau_{b}, \tau_{f}, S_{F}$ is not changed, the above formula is as follows:

$$
\delta h=\frac{K_{m}}{K_{m}+Q} \delta S
$$

This type is the base of feedback control system mathematical model. Same reason formula (6) can also be exported disturbance $\delta H, \delta K, \delta \mu$, and the relationship between the roll gap $\delta S$.

The thickness equation is substituted bouncing incremental form equation, the pressure equation is:

$$
\delta P=\frac{K_{m}}{K_{m}+Q}\left[\frac{\partial P}{\partial H} \delta H+Q\left(\delta S+\delta S_{F}\right)+\frac{\partial P}{\partial \tau_{b}} \delta \tau_{b}+\frac{\partial P}{\partial \tau_{f}}+\frac{\partial P}{\partial K} \delta K+\frac{\partial P}{\partial \mu} \delta \mu\right]
$$

This type visually indicates the impact of the rolling force on the control and disturbance amount.

\section{Tension equation}

In the rolling process, tension effects on the rolling member are due to the speed difference in the rolling longitudinal direction, making the different parts of rolling member bring out the relative displacement so that tensile stress is generated.

$$
T=A \times \tau
$$

Where $\mathrm{T}$ - the tension which is used on section $\mathrm{A}, \mathrm{N}$

$$
\begin{aligned}
& \text { A - cross-sectional area of strip, } \mathrm{m}^{2} \\
& \tau \text { - Average unit tensile stress, } N / \mathrm{m}^{2}
\end{aligned}
$$

Hooke's law shows that

$$
\tau=E \varepsilon
$$

where E-Modulus of the material elasticity , $\varepsilon$-Elastic strain

Taking two points a and $\mathrm{b}$ in a rolling, with the distance between two points is denoted by $l_{0}$, the velocity of point a and point $\mathrm{b}$ is respectively $V_{a}$ and $V_{b}$, and the displacement in the rolling length direction is $\mathrm{V} l$, then, 


$$
\begin{gathered}
\varepsilon=\frac{\mathrm{V} l}{l_{0}} \\
d \tau=E d \varepsilon=E d\left(\frac{\mathrm{V} l}{l_{0}}\right)=\frac{E}{L_{0}}\left(V_{b}-V_{a}\right) d t \\
\tau=\frac{E}{l_{0}} \int\left(V_{b}-V_{a}\right) d t \\
T=A \frac{E}{l_{0}} \int\left(V_{b}-V_{a}\right) d t
\end{gathered}
$$

Equation (13) shows that, in the specific rolling conditions, A, E, and $l_{0}$ are constant, the tension which is caused during the rolling process is entirely due to the generation of speed difference between point a and point $b$. In most of actual production, the approach of direct control tension is widely used, scilicet we use tonometer to measure the actual tension and make it as a feedback signal so that keep tension constant.

\section{The Establishment of Hydraulic AGC System}

\section{A. Forward feedback mathematical model of AGC system}

Thickness deviation $\partial H$ is obtained by forward feedback AGC system, according to the rolling entrance thickness gauge, to eliminate the exit thickness deviation by controlling the hydraulic cylinder and roll gap. The deviation signal of gauge should be sent in the shift register because of the space between gauge and roll gap in the controlling process. $\partial H$ was delayed for a short time, then the roll gap, entering the frame work should be under control to achieve optimal control effect. The advantage of the method is that it can overcome the delay in time; however, it belongs to open-circuit control range.

$\delta K$ cannot be actually obtained from forward feedback control, and we assume that $\delta K=0, \delta \tau_{b}=0, \delta \tau_{f}=0, \delta \mu=0$ and $\delta S_{F}=0$, then the thickness control equation is:

$$
\delta h=\frac{1}{K_{m}+Q}\left(Q \delta H+K_{m} \delta S\right)
$$

To eliminate the exit deviation in advance, when taking various kinds of AGC systems into consideration, then we can conclude that:

$$
\delta S_{F F}=-(1+\alpha) G_{S F F} \frac{Q}{K_{m}} \delta H
$$

where, $\alpha$ is compensation coefficient, $G_{S F F}$ is the gain value, and $\delta H$ is the entrance thickness deviation.

\section{B. Mathematical model of monitoring AGC system}

The elimination of deviation is done by controlling the hydraulic cylinder and roll gap according to the thickness deviation obtained from entrance gauge of monitoring AGC 
system. The main task of the system is to deal with any slow drift in the production process and compensate some dynamic error, to ensure the accuracy of the system.

Assuming that the tensile force of other frame works is constant, and $\delta \mu=0$ when rolling speed is constant. If $\delta K=0, \delta H=0$, we can get thickness equation formula 6 ignoring the impact of $\delta S_{F}$ :

$$
\delta h=-\frac{1}{K_{m}+Q} K_{m} \delta S
$$

The screw down value of monitoring AGC is set when taking integrated function of AGC system, exit thickness gauge, and the small delay in time:

$$
\delta S_{F B}=-\frac{K_{m}+Q}{K_{m}} K_{P}\left(1+\frac{K_{I}}{s}\right) \nabla h^{\prime}
$$

where, $K_{M}=K_{m} /(1+\alpha), \delta S_{F B}$ is the adjustment of roll gap, $K_{m}$ is the stiffness of mill, $\alpha$ is adjustment coefficient, $K_{p}$ is proportional gain adjustment coefficient, $K_{I}$ is the integral gain adjustment coefficient, and $\Delta h$ is the compensation value of thickness deviation, and $s$ is Laplace operator.

\section{Mathematical model of flow control per second AGC system}

Flow control per second AGC is an advanced thickness control method equipped on the modern cold rolling production line, which can be applied on every framework in the group. The exit thickness of framework can be obtained if the entrance thickness, speed and the exit speed is already known. Comparison between the value above and target value is conducted to obtain the exit deviation and achieve elimination by controlling the roll gap and rolling speed. The algorithm is as follows:

$$
\begin{gathered}
\delta S_{M F}=-\frac{K_{M}+Q}{K_{M}} G_{S M F}\left(h_{m}-h\right) \\
\delta V_{M F}=\frac{K_{P}}{h}\left(1+\frac{K_{I}}{s}\right)\left(h_{m}-h\right)
\end{gathered}
$$

where, $\delta S_{M F}$ is the adjustment of roll gap, $G_{S M F}$ is the AGC gain of roll gap flow control per second, $\mathrm{Q}$ is the plastic coefficient of rolled metal, $\boldsymbol{h}_{\boldsymbol{m}}$ is the calculating thickness of flow control per second, $\delta V_{M F}$ is the adjustment of roll gap.

\section{Simulation and Analysis of Monitoring AGC System}

Author names and affiliations are to be centered beneath the title and printed in Times New Roman 12-point, non-boldface type. Multiple authors may be shown in a two or three-column format, with their affiliations below their respective names. Affiliations are centered below each author name, italicized, not bold. Include e-mail addresses if possible. Follow the author information by two blank lines before main text.

AGC is open-loop forward feedback control, the control accuracy completely depend on the calculation precision, but in the first frame, the plastic material stiffness coefficient Q can 
not be measured accurately, so the control accuracy is not very high, the combination with monitor AGC, which they learn from each other, can be used to l improve the accuracy.

Monitor AGC simulation model, the simulation results shown in Figure 7, the results show that the thickness error of the rolling is reduced from 46 before rolling to 5 after rolling so that there is $2 \mu \mathrm{m}$ steady-state error.

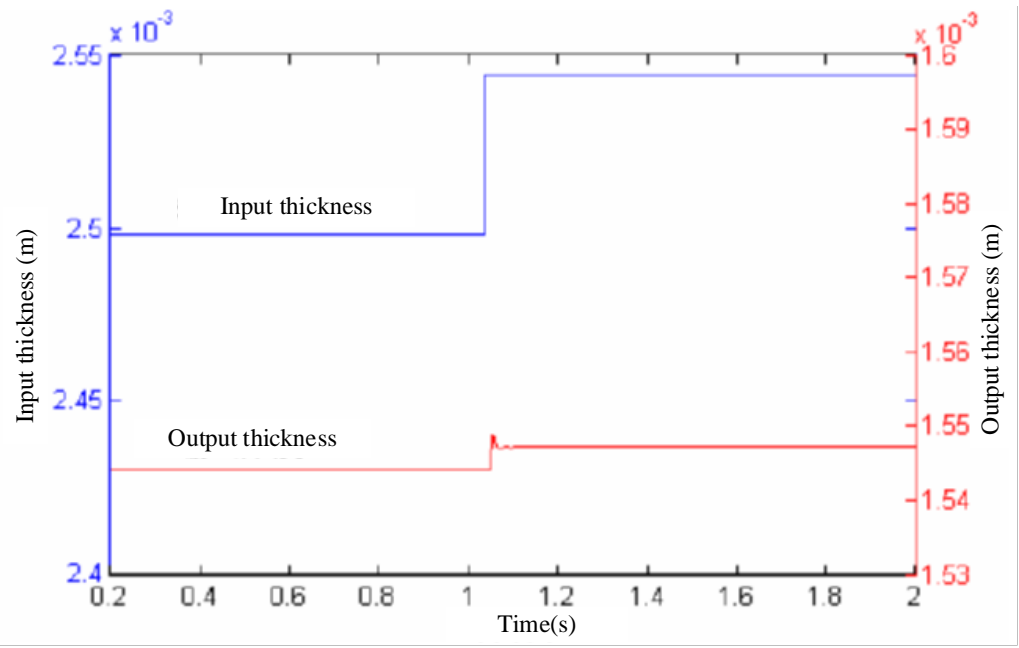

Figure 2. The Effect on Exports of Thickness Monitor AGC

\section{Conclusion}

The establishment of dynamic analysis model of cold rolling hydraulic AGC system has provided a complete and comprehensive analysis on the impact of various kinds of factors to the accuracy and thickness control in rolling process. The experimental results based on the dynamic analysis of proposed hydraulic AGC model is close to the theoretical data. Deeper research and dynamic simulation can be conducted in the whole cold rolling process through further research to achieve optimal control effect and has introductive significance to actual production.

\section{Acknowledgements}

The work was supported by Science and technology key project of science and technology department of Heilongjiang Province (GZ11A205).

\section{References}

[1] S. Yikang, "Model and control of hot continuous rolling of strip steel”, Metallurgical Industry Press, Beijing, (2002).

[2] G. Yingjie and Z. Jingyi, "Dynamic simulation of hydraulic AGC system of strip and plate rolling mills", Chinese Mechanical Engineering, vol. 9, no. 23, (1998).

[3] W. Guodong, L. Xianghua and W. Junsheng, “Automatic control of cold rolling thickness”, Steel rolling, vol. 3, no. 20, (2003).

[4] Y. Jie, “Mathematical model of rolling process”, Metallurgical Industry Press, Bei Jing, (1990).

[5] H. Zhijian and T. Zhaoqi, "Dynamic simulation method of error in hydraulic rolling mills", Metallurgical Device, vol. 4, no. 42, (2002).

[6] Y. Jingming and S. Weigong, "Dynamic mathematical model analysis and test of cold hydraulic rolling thickness control system”, Academic Journal of Yanshan University, vol. 1, no. 82, (1998).

[7] C. Zhangwei and Y. Shaochun, "On-line dynamic characteristic analysis on the hot hydraulic rolling screwdown control”, hydraulic and pneumatic theory, vol. 3, no. 45. (2003). 
International Journal of $\mathrm{u}$ - and e- Service, Science and Technology Vol.6, No.5 (2013) 